SEPTEMBER 1995, PAGES 401-421

\title{
COMPACTNESS FRAMEWORK AND CONVERGENCE \\ OF LAX-FRIEDRICHS AND GODUNOV SCHEMES \\ FOR A $2 \times 2$ NONSTRICTLY HYPERBOLIC SYSTEM \\ OF CONSERVATION LAWS
}

\author{
BY \\ BRUNO RUBINO \\ Scuola Normale Superiore, Piazza dei Cavalieri, Pisa, Italy
}

\begin{abstract}
A compactness framework and a convergence theorem for the LaxFriedrichs scheme and the Godunov scheme applied to the Cauchy problem for a $2 \times 2$ nonstrictly hyperbolic system of conservation laws are established. The existence of weak solutions is proved using the theory of compensated compactness of Tartar, Murat, DiPerna, and Serre.
\end{abstract}

1. Introduction. We are concerned here with the convergence of two types of approximations to a weak solution of the Cauchy problem for the following $2 \times 2$ system of conservation laws:

$$
\left\{\begin{array}{l}
u_{t}+\left(\left(\frac{1}{2}+n\right) u^{2}+f(v)\right)_{x}=0 \\
v_{t}+(u v)_{x}=0 \\
(u, v)_{\mid t=0}=\left(u_{0}, v_{0}\right)
\end{array}\right.
$$

where $n \in \mathbf{N}, f \in C^{2}(\mathbb{R})$ is an even and superlinear function such that $f(v)=$ $k v^{2 n}+O\left(|v|^{2 n+1}\right)$ for some $k \in \mathbb{R}^{+}$, and for all $v$ satisfying

$$
\begin{aligned}
f^{\prime}(v) v>0 & \text { for } v \neq 0 \\
f^{\prime \prime}(v)>0 & \text { for } v \neq 0 .
\end{aligned}
$$

In a previous paper [31] we investigated the convergence of vanishing viscosity approximation to a weak solution of the Cauchy problem (1.1) by employing some of the ideas developed by Kan [19] in his doctoral thesis for the case wherein $f(v)=\frac{1}{2} v^{2}$. Systems of this type have been numerically studied in connection with oil reservoir engineering models in [17] and [18], and they have also been investigated in some problems of mathematical physics $[1,2,8,20,32]$.

Here we prove the convergence of two conservative finite-difference schemes, namely the Lax-Friedrichs scheme and the Godunov scheme. A complete account of

Received May 3, 1992.

1991 Mathematics Subject Classification. Primary 35L65, 76L05.

Key words and phrases. Conservation laws, entropy, Goursat problem, invariant region, rarefaction wave, Riemann function, Riemann invariant, Riemann problem, shock wave, umbilical point.

(C) 1995 Brown University 
the numerical literature on these approximations and convergence goes beyond the scope of this paper; however, we wish to mention [1, 2, 5, 7, 11, 13, 14, 22, 27].

The method of compensated compactness is the main tool in proving the convergence of the Lax-Friedrichs and the Godunov approximations to a weak solution for (1.1) and we refer to the classical papers of Tartar [36], Murat [29], and DiPerna $[6,7,8,9]$ for the most important ideas in the general theory. Moreover, some important contributions are also due to Chen, Ding and Luo [1], Chen [2], and Serre [34].

In order to use the compensated compactness method we need to establish a uniform $L^{\infty}$-bound for classical difference schemes. The only pointwise bounds currently available for the difference scheme for $2 \times 2$ systems are those derived by using invariant regions $([3,15,35])$.

Now we observe that the system (1.1) under the previous assumptions fails to be strictly hyperbolic at the origin, where an isolated umbilical point occurs. Indeed, if we write the system (1.1) in the vector form

$$
\left\{\begin{array}{l}
\mathscr{U}_{t}+F(\mathscr{U})_{x}=0 \\
\mathscr{U}_{\mid t=0}=\mathscr{U}_{0}
\end{array}\right.
$$

by denoting $\mathscr{U}=(u, v)^{\mathrm{T}}, F(\mathscr{U})=\left(\left(\frac{1}{2}+n\right) u^{2}+f(v), u v\right)^{\mathrm{T}}$, then it is strictly hyperbolic when the two characteristic speeds, namely the eigenvalues $\lambda_{\mp}=\lambda_{\mp}(u, v)$, satisfy

$$
\lambda_{-}<\lambda_{+} \text {. }
$$

But in our case, defining

$$
G(u, v)=n^{2} u^{2}+v f^{\prime}(v),
$$

we get that the characteristic speeds of the problem are given by

$$
\lambda_{\mp}(u, v)=(1+n) u \mp \sqrt{G(u, v)}
$$

since $v f^{\prime}(v)>0$ for all $v \neq 0$, it follows that

$$
\lambda_{-}(u, v)<\lambda_{+}(u, v)
$$

for all $(u, v) \neq(0,0)$ and $\lambda_{-}(0,0)=\lambda_{+}(0,0)$.

Further, the system (1.1) is genuinely nonlinear if

$$
\nabla \lambda_{\mp} \cdot r_{\mp} \neq 0
$$

for all $(u, v)$, where $r_{\mp}$ are the right eigenvectors of $\lambda_{\mp}$.

In our case, letting

$$
g_{\mp}(u, v)=-n u \mp \sqrt{G(u, v)},
$$

we have $r_{\mp}(u, v)=\left(f^{\prime}(v), g_{\mp}(u, v)\right)^{\mathrm{T}}$; so we have

$$
\begin{array}{r}
\nabla \lambda_{\mp} \cdot r_{\mp}=\frac{1}{2 \sqrt{G(u, v)}}\left\{[(2 n+3) \sqrt{G(u, v)} \mp n(2 n-1) u] f^{\prime}(v)\right. \\
\left.+[\sqrt{G(u, v)} \pm n u] v f^{\prime \prime}(v)\right\}
\end{array}
$$


and the genuine nonlinearity fails along the $u$-axis: $\left\{(u, v) \in \mathbb{R}^{2}: v=0\right\}$.

A weak solution to (1.2) is a measurable function $\mathscr{U}=\mathscr{U}(x, t)$ such that, for all $\Phi \in C_{0}^{1}$, one has

$$
\int_{0}^{+\infty} \int_{-\infty}^{+\infty}\left(\mathscr{U}(x, t) \Phi_{t}+F(\mathscr{U}(x, t)) \Phi_{x}\right) d x d t+\int_{-\infty}^{+\infty} \mathscr{U}_{0}(x) \Phi(x, 0) d x=0 .
$$

An entropy-entropy flux pair of the system (1.2) is defined to be a pair of functions $\eta, q \in C^{1}\left(\mathbb{R}^{2}, \mathbb{R}\right)$ such that

$$
\nabla q=\nabla \eta \nabla F
$$

We say that a solution $\mathscr{U}(x, t)$ satisfies the entropy inequality in the sense of Krŭzkov [21] and Lax [25] (see also Hörmander [16]) if for any convex $\eta$ we have

$$
\eta\left(\mathscr{U}^{l}\right)_{t}+q\left(\mathscr{U}^{l}\right)_{x} \leq 0
$$

in $\mathscr{D}^{\prime}$.

Now let us recall [31] that the mechanical energy is given by

$$
\eta_{*}(u, v)=\frac{1}{2} u^{2}+\int_{0}^{v} \int_{0}^{y} \frac{f^{\prime}(z)}{z} d z d y
$$

which is a strictly convex entropy for (1.1): the corresponding flux is given by

$$
q_{*}(u, v)=\frac{2 n+1}{3} u^{3}+u v \int_{0}^{v} \frac{f^{\prime}(z)}{z} d z
$$

The entropy-entropy flux pair $\left(\eta_{*}, q_{*}\right)$ will play a fundamental role in getting the energy estimates, which will be given in a later section.

The plan of the paper is as follows. Section 2 summarizes the results on the existence of the invariant domain and the a priori bounds in $L^{\infty}$. For convenience of the reader, in Sec. 3 we shall recall the construction of Lax-Friedrich approximations and the Godunov approximations. In Sec. 4 we construct a special class of such entropies in order to study, in the final section, the behavior of approximation schemes and to establish that the $\mathrm{H}^{-1}$-condition and the other compactness frameworks require the theory of Tartar and DiPerna.

2. Invariant regions. In this section we discuss the construction of invariant regions for the system (1.2). We define the Riemann problem (1.2) with initial data of the form

$$
\mathscr{U}_{0}(x)= \begin{cases}\mathscr{U}_{-} & x<0, \\ \mathscr{U}_{+} & x>0 .\end{cases}
$$

The solution of the Riemann problem for a hyperbolic system of conservation laws was constructed by Lax in [23], and he proved that, when $\mathscr{U}_{-}$and $\mathscr{U}_{+}$are sufficiently close, there is a unique solution of the Riemann problem of a certain type (admissibility condition). Now we say that $\Sigma$ is invariant if, whenever $\mathscr{U}_{-}$and $\mathscr{U}_{+}$are in $\Sigma$ and the Lax solution $\mathscr{U}(x, t)$ of the corresponding Riemann problem exists, then $\mathscr{U}(x, t) \in \Sigma$ for all $(x, t)$. 
Let us recall that $\omega_{-} \in C^{1} \quad\left(\omega_{+}\right.$respectively $)$is a first (second) Riemann invariant for (1.2) if for all $(u, v)$

$$
\left(\nabla \omega_{-}(u, v)\right)^{\mathrm{T}} \cdot r_{+}(u, v)=0
$$

$\left(\left(\nabla \omega_{+}(u, v)\right)^{\mathrm{T}} \cdot r_{-}(u, v)=0\right)$. The integral curves $R_{\mp}$ of $r_{\mp}$ in the state space are respectively called the first and second rarefaction wave curves. So, in our case, a first (second) rarefaction curve satisfies the ordinary first-order differential equation

$$
\frac{d v}{d u}=\frac{g_{\mp}(u, v)}{f^{\prime}(v)} .
$$

It can be easily proved (see [31]) that the following properties hold.

THeOREM 2.1. For the Cauchy problem (1.1) the curves $R_{-}$are obtained by a mirror reflection of the curves $R_{+}$about the $v$-axis. Further, if we restrict to the $R_{+}$family, moreover

a) the $R_{+}$curves do not intersect the positive $u$-axis;

b) the $R_{+}$curves are in one-to-one correspondence with the points of the negative $u$-semiaxis;

c) each one of the $R_{+}$curves is confined to the upper half plane and tends to $(0,0)$ as $v \rightarrow 0$

d) the half line $\{(u, v): u<0, v=0\}$ is itself an $R_{+}$curve;

e) every $R_{+}$curve that does not stay on the $u$-axis tends to $\infty$ as $u \rightarrow+\infty$.

Hence we can conclude that an $R_{+}$curve either starts from the origin and stays on the $u$-axis going to infinity to the right or it intersects the negative $u$-axis and goes to infinity to the right.

Finally, for $(1.1)$ we can construct Riemann invariants $\omega_{-}, \omega_{+}$so that

$$
\begin{gathered}
\omega_{-}(u, v) \leq 0 \leq \omega_{+}(u, v), \\
\omega_{-}(u, 0)=\left\{\begin{array}{ll}
u & u \leq 0 \\
0 & u>0
\end{array}, \quad \omega_{+}(u, 0)= \begin{cases}0 & u<0 \\
u & u \leq 0\end{cases} \right.
\end{gathered}
$$

and

$$
\begin{aligned}
& \frac{1}{f^{\prime}(v)} \frac{\partial \omega_{-}}{\partial v}<0, \\
& \frac{1}{f^{\prime}(v)} \frac{\partial \omega_{+}}{\partial v}>0 .
\end{aligned}
$$

But, as a consequence of the results of Chueh, Conley, and Smoller [3], Hoff [15] proves the following result.

Proposition. A region $\Sigma$ is invariant for (1.2)-(2.1) if at each point $\mathscr{U}_{0}$ of $\partial \Sigma$ one of the left eigenvectors $l_{\mp}$ is parallel to the normal to $\partial \Sigma$.

So, as was proved in [31] for the parabolic system, we can now conclude that for all $c>0$ the regions $\left\{\Sigma_{c}\right\}$ given by

$$
\Sigma_{c}=\left\{(u, v): \omega_{-}+c \geq 0\right\} \cap\left\{(u, v): \omega_{+}-c \leq 0\right\} \cap\{(u, v): v \geq 0\}
$$


form a family of invariant domains. Further, as we will easily prove in Sec. 4, if a convex set $\Sigma$ is invariant for solutions of the Riemann problem, then it remains invariant for the Lax-Friedrichs and Godunov schemes. Then the construction is complete.

3. Lax-Friedrichs approximations and Godunov approximations. In this section we consider Lax-Friedrichs and Godunov schemes for the problem (1.1) and study their behavior. On the upper half plane $\mathbb{R}_{x} \times \mathbb{R}_{t}^{+}$we have the grid

$$
\{(j l, i h): j \in \mathbf{Z}, i \in \mathbf{N}\}
$$

where $h, l \in \mathbb{R}^{+} \backslash\{0\}$ are the time step length and the space step length, respectively, that satisfy the $C F L$ condition

$$
\max _{\mp}\left(\sup _{\mathbb{R}_{x} \times[0, T]}\left|\lambda_{\mp}\left(\mathscr{U}^{l}\right)\right|\right)<\frac{l}{h} \leq M
$$

for any given $T>0$. Since we assume that $v^{l}(x, t) \geq 0$, it is possible to construct $\mathscr{U}^{l}(x, t)$.

Let

$$
J_{i}=\{j \in \mathbf{Z}: i+j \in \mathbf{2 Z}\}
$$

On the rectangle

$$
\left\{(x, t) \in \mathbb{R}_{x} \times \mathbb{R}_{t}^{+}:(j-1) l<x<(j+1) l, 0 \leq t<h,(j-1) \in 2 \mathbf{Z}\right\},
$$

we define $\mathscr{U}^{l}(x, t)=\left(u^{l}, v^{l}\right)(x, t)$ as the solution of the Riemann problem

$$
\left\{\begin{array}{l}
u_{t}+\left(\left(\frac{1}{2}+n\right) u^{2}+f(v)\right)_{x}=0, \\
v_{t}+(u v)_{x}=0, \\
(u, v)_{\mid t=0}= \begin{cases}\left(u_{0}^{l}((j-1) l), v_{0}^{l}((j-1) l)\right) & x<j l, \\
\left(u_{0}^{l}((j+1) l), v_{0}^{l}((j+1) l)\right) & x>j l,\end{cases}
\end{array}\right.
$$

where $\mathscr{U}_{0}^{l}(x)=\mathscr{U}_{0}(x) \chi_{\left[-\frac{1}{\dagger},+\frac{1}{1}\right]}(x)$. Let us now define

$$
\mathscr{U}_{j}^{1}=\frac{1}{2 l} \int_{(j-1) l}^{(j+1) l} \mathscr{U}^{l}\left(x, h^{-}\right) d x .
$$

If we have defined $\mathscr{U}^{l}$ for $t<i h$, we define $\mathscr{U}^{l}$ on the rectangle

$$
\left\{(x, t) \in \mathbb{R}_{x} \times \mathbb{R}_{t}^{+}:(j-1) l<x<(j+1) l, i h<t<(i+1) h, j \in J_{i}\right\}
$$

as the solution of the Riemann problem

$$
\left\{\begin{array}{l}
u_{t}+\left(\left(\frac{1}{2}+n\right) u^{2}+f(v)\right)_{x}=0 \\
v_{t}+(u v)_{x}=0 \\
(u, v)_{\mid t=i h}= \begin{cases}\left(u_{j-1}^{i}, v_{j-1}^{i}\right) & x<j l, \\
\left(u_{j+1}^{i}, v_{j+1}^{i}\right) & x>j l .\end{cases}
\end{array}\right.
$$


Finally, we define the Lax-Friedrichs scheme as the scheme

$$
\mathscr{U}_{j}^{i+1}=\frac{1}{2 l} \int_{(j-1) l}^{(j+1) l} \mathscr{U}^{l}\left(x,(i+1) h^{-}\right) d x .
$$

In the same way, on the rectangle

$$
\left\{(x, t) \in \mathbb{R}_{x} \times \mathbb{R}_{t}^{+}: j l<x<(j+1) l, 0 \leq t<h\right\},
$$

we define $\mathscr{U}^{l}(x, t)=\left(u^{l}(x, t), v^{l}(x, t)\right)$ as the solution of the Riemann problem

$$
\left\{\begin{array}{l}
u_{t}+\left(\left(\frac{1}{2}+n\right) u^{2}+f(v)\right)_{x}=0, \\
v_{t}+(u v)_{x}=0, \\
(u, v)_{\mid t=0}= \begin{cases}\left(u_{0}^{l}(j l), v_{0}^{l}(j l)\right) & x<\left(j+\frac{1}{2}\right) l, \\
\left(u_{0}^{l}(j l), v_{0}^{l}(j l)\right. & x>\left(j+\frac{1}{2}\right) l,\end{cases}
\end{array}\right.
$$

where $\mathscr{U}_{0}^{l}(x)=\mathscr{U}_{0}(x) \chi_{\left[-\frac{1}{t},+\frac{1}{l}\right]}(x)$. Let us now define

$$
\mathscr{U}_{j}^{1}=\frac{1}{l} \int_{\left(j-\frac{1}{2}\right) l}^{\left(j+\frac{1}{2}\right) l} \mathscr{U}^{l}\left(x, h^{-}\right) d x .
$$

If we have defined $\mathscr{U}^{l}$ for $t<i h$, we define $\mathscr{U}^{l}$ on the rectangle

$$
\left\{(x, t) \in \mathbb{R}_{x} \times \mathbb{R}_{t}^{+}: j l<x<(j+1) l, i h<t<(i+1) h\right\}
$$

to be the solution of the Riemann problem

$$
\left\{\begin{array}{l}
u_{t}+\left(\left(\frac{1}{2}+n\right) u^{2}+f(v)\right)_{x}=0, \\
v_{t}+(u v)_{x}=0, \\
(u, v)_{\mid t=i h}= \begin{cases}\left(u_{j}^{i}, v_{j}^{i}\right) & x<\left(j+\frac{1}{2}\right) l, \\
\left(u_{j+1}^{i}, v_{j+1}^{i}\right) & x>\left(j+\frac{1}{2}\right) l .\end{cases}
\end{array}\right.
$$

As before, we define the Godunov scheme as the scheme

$$
\mathscr{U}_{j}^{i+1}=\frac{1}{l} \int_{\left(j-\frac{1}{2}\right) l}^{\left(j+\frac{1}{2}\right) l} \mathscr{U}^{l}\left(x,(i+1) h^{-}\right) d x .
$$

This completes the construction of the two finite-difference schemes.

4. Entropies. We shall construct a special class of entropies in order to establish the convergence for the Lax-Friedrichs and Godunov approximation to problem (1.1).

Let us notice that

$$
T:(u, v) \rightarrow\left(\omega_{-}, \omega_{+}\right)
$$

is a one-to-one map defining a change of coordinates; so the Riemann invariants $\left(\omega_{-}, \omega_{+}\right)$can be assumed as a new system of coordinates. Here the characteristic curves for the entropy equations are the straight lines parallel to the coordinate axes.

Now we consider the Goursat problem associated to the equation

$$
\frac{\partial^{2} \eta}{\partial \omega_{-} \partial \omega_{+}}+\frac{1}{\lambda_{+}(\omega)-\lambda_{-}(\omega)}\left(\frac{\partial \lambda_{+}(\omega)}{\partial \omega_{-}} \frac{\partial \eta}{\partial \omega_{+}}-\frac{\partial \lambda_{-}(\omega)}{\partial \omega_{+}} \frac{\partial \eta}{\partial \omega_{-}}\right)=0
$$


with data

$$
\begin{aligned}
& \eta\left(\omega_{-}, \omega_{+}^{*}\right)=\theta_{-}\left(\omega_{-}\right), \\
& \eta\left(\omega_{-}^{*}, \omega_{+}\right)=\theta_{+}\left(\omega_{+}\right),
\end{aligned}
$$

where $\theta_{-}$and $\theta_{+}$are given functions. We recall the following classical results [36]. THEOREM. The solution of the Goursat problem (4.1)-(4.2), in any domain away from the umbilical point and from the $\omega_{+}$-axis, has the same regularity as the initial data.

As a consequence to the singularity of the coefficients of (4.1) in the umbilical point, we shall consider only particular Goursat data, namely

$$
\begin{aligned}
& \theta_{-}\left(\omega_{-}\right)=0 \quad \text { in } \omega_{-} \leq \omega_{-}^{*}, \\
& \theta_{+}\left(\omega_{+}\right) \equiv 0
\end{aligned}
$$

and particular constants, namely

$$
\begin{aligned}
& \omega_{-}^{*}<0, \\
& \omega_{+}^{*}=0 .
\end{aligned}
$$

In this way, the solution will have some particular vanishing properties and, in particular,

$$
\eta(\omega)=0 \text { for } \omega_{-} \leq \omega_{-}^{*} .
$$

The technical complications that we meet are mainly due to our incapacity to write explicitly the change of coordinate $T$. However, as we easily see, there exists a Riemann invariant coordinate system $\left(\omega_{-}, \omega_{+}\right)$, such that

$$
\begin{aligned}
\frac{\partial u}{\partial \omega_{-}} & =\frac{1}{2}, \\
\frac{\partial v}{\partial \omega_{-}} & =\frac{-n u-\sqrt{n^{2} u^{2}+v f^{\prime}(v)}}{2 f^{\prime}(v)}, \\
\frac{\partial u}{\partial \omega_{+}} & =\frac{1}{2}, \\
\frac{\partial v}{\partial \omega_{+}} & =\frac{-n u+\sqrt{n^{2} u^{2}+v f^{\prime}(v)}}{2 f^{\prime}(v)} .
\end{aligned}
$$

In this way we can estimate the asymptotic behaviour of the coefficients of the firstorder term in Eq. (4.1). One has the following result [31].

LemmA 4.1. With the previous assumptions on the Riemann invariant $\left(\omega_{-}, \omega_{+}\right)$, the eigenvalues $\lambda_{ \pm}(\omega)$ satisfy the properties:

a)

$$
\begin{aligned}
& \lim _{|\omega| \rightarrow 0} \frac{\partial \lambda_{+}(\omega)}{\partial \omega_{-}}=\frac{1}{2}, \\
& \lim _{|\omega| \rightarrow 0} \frac{\partial \lambda_{-}(\omega)}{\partial \omega_{+}}=\frac{1}{2}
\end{aligned}
$$


b) in a neighbourhood of the umbilical point one has

$$
\lambda_{+}(\omega)-\lambda_{-}(\omega)=n\left(\omega_{+}-\omega_{-}\right)+O(|\omega|) .
$$

Proof. By using (4.4), we have

$$
\frac{\partial \lambda_{+}}{\partial \omega_{-}}(\omega)=\frac{1}{2}+\frac{1}{2}\left(n-\frac{v f^{\prime \prime}(v)+f^{\prime}(v)}{2 f^{\prime}(v)}\right)\left(1+\frac{n u}{\sqrt{n^{2} u^{2}+v f^{\prime}(v)}}\right)
$$

and

$$
\frac{\partial \lambda_{-}}{\partial \omega_{+}}(\omega)=\frac{1}{2}+\frac{1}{2}\left(n-\frac{v f^{\prime \prime}(v)+f^{\prime}(v)}{2 f^{\prime}(v)}\right)\left(1-\frac{n u}{\sqrt{n^{2} u^{2}+v f^{\prime}(v)}}\right) .
$$

On the other hand,

$$
\lambda_{+}(\omega)-\lambda_{-}(\omega)=2 \sqrt{n^{2} u^{2}+v f^{\prime}(v)} ;
$$

hence,

$$
\begin{aligned}
& \frac{\partial}{\partial \omega_{-}}\left(\lambda_{+}(\omega)-\lambda_{-}(\omega)\right)=-\frac{v f^{\prime \prime}(v)+f^{\prime}(v)}{2 f^{\prime}(v)}+\frac{n u}{\sqrt{n^{2} u^{2}+v f^{\prime}(v)}}\left(n-\frac{v f^{\prime \prime}(v)+f^{\prime}(v)}{2 f^{\prime}(v)}\right), \\
& \frac{\partial}{\partial \omega_{+}}\left(\lambda_{+}(\omega)-\lambda_{-}(\omega)\right)=+\frac{v f^{\prime \prime}(v)+f^{\prime}(v)}{2 f^{\prime}(v)}+\frac{n u}{\sqrt{n^{2} u^{2}+v f^{\prime}(v)}}\left(n-\frac{v f^{\prime \prime}(v)+f^{\prime}(v)}{2 f^{\prime}(v)}\right) .
\end{aligned}
$$

The result is achieved by taking into account that

$$
\lim _{v \rightarrow 0} \frac{v f^{\prime \prime}(v)+f^{\prime}(v)}{2 f^{\prime}(v)}=n .
$$

Now we notice that for our problem (4.1)-(4.2) the following Riemann representation formula (see [4, pp. 449/461]) holds:

$$
\begin{aligned}
\eta(\omega)=\int_{\omega_{-}^{*}}^{\omega_{-}} \mathscr{R}\left(t, 0, \omega_{-}, \omega_{+}\right) & \left(\frac{\partial \eta}{\partial \omega_{-}}\left(t, \omega_{+}\right)\right. \\
& \left.+\frac{\eta\left(t, \omega_{+}\right)}{\lambda_{+}\left(t, \omega_{+}\right)-\lambda_{-}\left(t, \omega_{+}\right)} \frac{\partial \lambda_{+}}{\partial \omega_{-}}\left(t, \omega_{+}\right)\right)_{\mid \omega_{+}=0} d t \\
= & \int_{\omega_{-}^{*}}^{\omega_{-}} \mathscr{R}\left(t, 0, \omega_{-}, \omega_{+}\right)\left(\theta_{-}^{\prime}(t)+\frac{\theta(t)}{\lambda_{+}(t, 0)-\lambda_{-}(t, 0)} \frac{\partial \lambda_{+}}{\partial \omega_{-}}(t, 0)\right) d t
\end{aligned}
$$

where $\mathscr{R}=\mathscr{R}\left(t, s, \omega_{-}, \omega_{+}\right)$is the Riemann function. Since $\theta_{-}$verifies the hypothesis (4.3), the singularity of $\mathscr{R}$ does not depend on $t$. Moreover, $\eta$ is regular on the half plane $\omega_{-}<k<0$ for all $k$. It is not difficult to show (see [19]) that it is sufficient to focus on the behaviour of $\eta$ on the square

$$
\mathscr{Q}_{\varepsilon}=\left\{\omega:-\varepsilon \leq \omega_{-} \leq 0 \leq \omega_{+} \leq \varepsilon\right\}
$$


for some $\varepsilon<\delta$; on the other hand, $\left(\omega_{+}-\omega_{-}\right) \mathscr{R}\left(t, 0, \omega_{-}, \omega_{+}\right)$is regular for all $t \in\left[\omega_{-}^{*},-\delta\right]$. Here we have, from (4.3),

$$
\eta(\omega)=\int_{\omega_{-}^{*}}^{-\delta} \mathscr{R}\left(t, 0, \omega_{-}, \omega_{+}\right)\left(\theta_{-}^{\prime}(t)+\frac{\theta_{-}(t)}{\lambda_{+}(t, 0)-\lambda_{-}(t, 0)} \frac{\partial \lambda_{+}}{\partial \omega_{-}}(t, 0)\right) d t .
$$

By the regularity of the Riemann function, using the methods of [19], we can write $\mathscr{R}$ as a sum of a regular part and a singular part given by

$$
\frac{r(\omega)}{\omega_{+}-\omega_{-}} \sigma(t)
$$

where $r$ is a regular function. If we now impose that

$$
\int_{\omega_{-}^{*}}^{-\delta} \sigma(t)\left(\theta_{-}^{\prime}(t)+\frac{\theta_{-}(t)}{\lambda_{+}(t, 0)-\lambda_{-}(t, 0)} \frac{\partial \lambda_{+}}{\partial \omega_{-}}(t, 0)\right) d t=0,
$$

the function $\eta$ is continuous everywhere. In a similar way we can investigate the higher-order derivatives of $\eta$, by imposing the additional restriction of the initial datum $\theta_{-}$. So it is possible to conclude with the following

Theorem 4.2. For the Goursat problem (4.1)-(4.2) there exists a data $\theta_{-}$, vanishing when $-\delta \leq \omega_{-} \leq 0$ for some $\delta>0$, such that the solution $\eta$ and its derivatives with respect to $\left(\omega_{-}, \omega_{+}\right)$up to the second order are bounded on bounded sets in the state space $\left(\omega_{-}, \omega_{+}\right)$.

The solution just found is said, following Serre [34], to be an east-type entropy with limit $\omega_{-}^{*}$. Similar theorems are true for entropy of west type with limit $\omega_{-}^{*}$ and of south or nord type with limit $\omega_{+}^{*}$.

These entropies will be used in the following section.

5. Strong convergence. Here we describe the limit behavior of the approximate solution $\mathscr{U}^{l}(x, t)$ generated from the Lax-Friedrichs scheme to the system (1.1). Then we apply the compensated compactness theory and establish strong convergence.

We shall now prove a compactness theorem by using the methods of DiPerna [6, 7] and Chen [1, 2]. The exposition follows strictly [1, 2].

THEOREM 5.1. Let $\left\{\mathscr{U}^{l}(x, t)\right\}$ be a sequence of approximate solutions generated by either the Lax-Friedrichs scheme or the Godunov scheme and let $\mathscr{U}_{0}(x)$ be the initial data satisfying the following hypothesis:

$$
\left\{\begin{array}{l}
\left|u_{0}(x)\right| \leq M, \\
0 \leq v_{0}(x) \leq M, \\
\int_{\mathbb{R}}\left(\eta_{*}\left(\mathscr{U}_{0}(x)\right)-\eta_{*}(\overline{\mathscr{U}})-\nabla \eta_{*}(\overline{\mathscr{U}})\left(\mathscr{U}_{0}(x)-\overline{\mathscr{U}}\right)\right) d x \leq M
\end{array}\right.
$$

for some constant state $\overline{\mathscr{U}}$, some constant $M>0$, and the mechanical energy (1.5). Then

i) there exists a constant $c>0$ such that

$$
\left|u^{l}(x, t)\right| \leq c, \quad 0 \leq v^{l}(x, t) \leq c \quad \text { a.e.; }
$$


ii) for all entropy pairs $(\eta, q)$, the measure

$$
\eta\left(\mathscr{U}^{l}\right)_{t}+q\left(\mathscr{U}^{l}\right)_{x}
$$

lies in a compact subset of $H_{\text {loc }}^{-1}$.

Proof. We limit ourselves to proving the result only for the Lax-Friedrichs scheme; the argument is similar in the other case. Without loss of generality, we may suppose

$$
\int_{\mathbb{R}_{x}} \eta_{*}\left(\mathscr{U}_{0}(x)\right) d x<+\infty
$$

otherwise, it would be sufficient to introduce a normalized entropy pair

$$
\begin{aligned}
& \tilde{\eta}_{*}(\mathscr{U})=\eta_{*}(\mathscr{U})-\nabla \eta_{*}(\overline{\mathscr{U}})(\mathscr{U}-\overline{\mathscr{U}}), \\
& \tilde{q}_{*}(\mathscr{U})=q_{*}(\mathscr{U})-\nabla \eta_{*}(\overline{\mathscr{U}})(F(\mathscr{U})-F(\overline{\mathscr{U}})) .
\end{aligned}
$$

First, we notice that the regions $\Sigma_{c}$ found in Sec. 2 are convex sets and invariant regions of the Riemann problem (3.1). So, if $\left\{\mathscr{U}(x, \cdot): x \in\left[x_{1}, x_{2}\right]\right\} \subset \Sigma_{c}$, then

$$
\frac{1}{x_{2}-x_{1}} \int_{x_{1}}^{x_{2}} \mathscr{U}(x, \cdot) d x \in \Sigma_{c} \text {. }
$$

Therefore, $\Sigma_{c}$ are invariant domains of the approximations, and the result (5.2) holds.

To arrive at (5.3), let $(\eta, q)$ be an entropy-entropy flux pair for the system (1.1) and $\mathscr{U}^{l}$ an approximate solution as constructed before. We give a representation formula for the measure (5.3) in order to show that it lies in a compact subset of $H_{\text {loc }}^{-1}$. Using Green's formula, for any $\Phi \in C_{0}^{1}\left(\mathscr{S}_{T}\right)$ with

$$
\mathscr{S}_{T}=\left\{(x, t) \in \mathbb{R}_{x} \times \mathbb{R}_{t}^{+}: 0 \leq t \leq T=m h\right\},
$$

we can write the entropy identity in the form

$$
\int_{\mathscr{S}_{T}} \int\left(\eta\left(\mathscr{U}^{l}\right) \Phi_{t}+q\left(\mathscr{U}^{l}\right) \Phi_{x}\right) d x d t=M(\Phi)+L(\Phi)+\Sigma(\Phi)
$$

where

$$
\begin{gathered}
M(\Phi)=\int_{\mathbb{R}_{x}} \Phi(x, T) \eta\left(\mathscr{U}^{l}(x, T)\right) d x-\int_{\mathbb{R}_{x}} \Phi(x, 0) \eta\left(\mathscr{U}^{l}(x, 0)\right) d x, \\
\Sigma(\Phi)=\int_{0}^{T} \sum_{\mathscr{S}}\{\sigma[\eta]-[q]\} \Phi(x(t), t) d t
\end{gathered}
$$

where $\mathscr{S}$ denotes the set of all shock waves in $\mathscr{U}^{l}$ at the fixed time $t, \sigma$ denotes the propagating speed of the shock wave, and $[f]$ denotes the jump of the function $f\left(\mathscr{U}^{l}(x, t)\right)$ across the shock wave $(x(t), t)$ from left to right, namely, $[f]=f\left(\mathscr{U}^{l}\left(x(t)^{+}, t\right)\right)-f\left(\mathscr{U}^{l}\left(x(t)^{-}, t\right)\right)$.

Finally, we decompose the interface error $L(\Phi)$ as follows:

$$
L(\Phi)=L_{1}(\Phi)+L_{2}(\Phi)
$$


where (for the Lax-Friedrichs scheme)

$$
\begin{aligned}
& L_{1}(\Phi)=\sum_{j+i \in 2 \mathrm{Z}} \boldsymbol{\Phi}_{j}^{i} \int_{(j-1) l}^{(j+1) l}\left[\eta_{j}^{i}\right] d x, \\
& L_{2}(\boldsymbol{\Phi})=\sum_{j+i \in 2 \mathbf{Z}} \int_{(j-1) l}^{(j+1) l}\left[\eta_{j}^{i}\right]\left(\Phi-\boldsymbol{\Phi}_{j}^{i}\right) d x,
\end{aligned}
$$

where $\left[\eta_{j}^{i}\right]=\eta\left(\mathscr{U}_{-}^{i}\right)-\eta\left(\mathscr{U}_{j}^{i}\right), \mathscr{U}_{-}^{i}=\mathscr{U}^{l}\left(x, i h^{-}\right)$, and $\Phi_{j}^{i}=\Phi(j l, i h)$.

Now we notice that $\mathscr{U}^{l}$ has compact support in the strip $\mathscr{S}_{T}$; therefore we may substitute, in the equality $(5.4),(\eta, q)=\left(\eta_{*}, q_{*}\right)$ and $\Phi(x) \equiv 1$. So we obtain

$$
0=M(1)+L(1)+\Sigma(1)
$$

from which

$$
\begin{aligned}
& \sum_{j+i \in 2 \mathbf{Z}} \int_{(j-1) l}^{(j+1) l}\left[\eta_{j}^{i}\right] d x+\int_{0}^{T} \sum_{\mathscr{S}}\left\{\sigma\left[\eta_{*}\right]-\left[q_{*}\right]\right\} d t \\
& \quad=\int_{\mathbb{R}_{x}} \eta_{*}\left(\mathscr{U}^{l}(x, 0)\right) d x-\int_{\mathbb{R}_{x}} \eta_{*}\left(\mathscr{U}^{l}(x, T)\right) d x \leq \int_{\mathbb{R}_{x}} \eta_{*}\left(\mathscr{U}^{l}(x, 0)\right) d x \leq \text { const. }
\end{aligned}
$$

On the other hand,

$$
\begin{aligned}
& \sum_{j+i \in 2 \mathbf{Z}} \int_{(j-1) l}^{(j+1) l}\left[\eta_{j}^{i}\right] d x \\
& \quad=\sum_{j+i \in 2 \mathbf{Z}} \int_{(j-1) l}^{(j+1) l} d x \int_{0}^{1}(1-\theta)\left(\mathscr{U}_{-}^{i}-\mathscr{U}_{j}^{i}\right) \nabla^{2} \eta_{*}\left(\mathscr{U}_{j}^{i}+\theta\left(\mathscr{U}_{-}^{i}-\mathscr{U}_{j}^{i}\right)\right)\left(\mathscr{U}_{-}^{i}-\mathscr{U}_{j}^{i}\right) d \theta
\end{aligned}
$$

We observe, in passing, that if $\eta$ is convex, then the entropy inequality

$$
\sigma[\eta]-[q] \geq 0
$$

is satisfied across the shock waves. So we obtain from (5.5)-(5.6) that

$$
\begin{gathered}
\int_{0}^{T} \sum_{\mathscr{S}}\left\{\sigma\left[\eta_{*}\right]-\left[q_{*}\right]\right\} d t \leq \mathrm{const}, \\
\sum_{j+i \in 2 \mathrm{Z}} \int_{(j-1) l}^{(j+1) l} d x \int_{0}^{1}(1-\theta)\left(\mathscr{U}_{-}^{i}-\mathscr{U}_{j}^{i}\right) \nabla^{2} \eta_{*}\left(\mathscr{U}_{j}^{i}+\theta\left(\mathscr{U}_{-}^{i}-\mathscr{U}_{j}^{i}\right)\right)\left(\mathscr{U}_{-}^{i}-\mathscr{U}_{j}^{i}\right) d \theta \leq \mathrm{const} .
\end{gathered}
$$

To show that the measure (5.3) lies in a compact subset of $H_{\text {loc }}^{-1}$, it is sufficient to show that $M, L$, and $\Sigma$ lie in a compact subset of $W_{\text {loc }}^{-1,1}$. Hence (5.3) lies in a compact subset of $W_{\mathrm{loc}}^{-1,1}$ and in a bounded subset of $W^{-1, \infty}$.

First we remark that [6]:

$$
\begin{aligned}
|M(\Phi)| & \leq \text { const }|\Phi|_{\infty}, \\
|\Sigma(\Phi)| & \leq \text { const }|\Phi|_{\infty} ;
\end{aligned}
$$

hence, $M$ and $\Sigma$ lie in a bounded set of $C^{*}$ and in a compact subset of $W_{\text {loc }}^{-1,1}$. 
We complete the proof by showing that $L_{1}$ and $L_{2}$ satisfy the estimates

$$
\begin{aligned}
& \left|L_{1}(\Phi)\right| \leq \text { const }|\Phi|_{\infty}, \\
& \left|L_{2}(\Phi)\right| \leq \text { const } l^{\beta}|\Phi|_{C^{0, \alpha}}
\end{aligned}
$$

for appropriate $\alpha$ and $\beta$ : by the Sobolev imbedding theorem

$$
\left|L_{2}(\Phi)\right| \leq \text { const } l^{\beta}|\Phi|_{W^{1, p}}
$$

for an appropriate $p$ and an appropriate constant depending on the support of $\Phi$. Hence $L_{2}$ lies in a compact subset of $W_{\text {loc }}^{-1, q}$. First of all we notice that

$$
\begin{aligned}
\left|L_{1}(\Phi)\right| \leq & \|\Phi\|_{C^{0}} \sum_{j+i \in 2 Z}\left|\int_{(j-1) l}^{(j+1) l}\left[\eta_{j}^{i}\right] d x\right| \\
\leq & \text { const }\|\Phi\|_{C^{0}} \sum_{j+i \in 2 Z} \int_{(j-1) l}^{(j+1) l} d x \int_{0}^{1}(1-\theta) \nabla^{2} \eta_{*} \\
& \times\left(\mathscr{U}_{j}^{i}+\theta\left(\mathscr{U}_{-}^{i}-\mathscr{U}_{j}^{i}\right)\right)\left(\mathscr{U}_{-}^{i}-\mathscr{U}_{j}^{i}, \mathscr{U}_{-}^{i}-\mathscr{U}_{j}^{i}\right) d \theta \\
\leq & \text { const }\|\Phi\|_{C^{0}} .
\end{aligned}
$$

Hence,

$$
\left\|M+L_{1}+\Sigma\right\|_{C_{0}^{*}} \leq \text { const }
$$

and, by the Sobolev imbedding theorem, $M+L_{1}+\Sigma$ lies in a compact subset of $W^{-1, q_{0}}$, where $1<q_{0}<2$. On the other hand, $\Phi \in C^{\alpha}(\Omega), \frac{1}{2}<\alpha<1$, and we have

$$
\begin{aligned}
\left|L_{2}(\Phi)\right| & \leq \sum_{j+i \in 2 \mathrm{Z}} \int_{(j-1) l}^{(j+1) l}|\Phi(x, i h)-\Phi(j l, i h)|\left|\left[\eta_{j}^{i}\right]\right| d x \\
& \leq \sum_{i}\left(\sum_{j} \int_{(j-1) l}^{(j+1) l}|\Phi(x, i h)-\Phi(j l, i h)|^{2} d x\right)^{1 / 2}\left(\sum_{j} \int_{(j-1) l}^{(j+1) l}\left|\left[\eta_{j}^{i}\right]\right|^{2} d x\right)^{1 / 2} .
\end{aligned}
$$

Now we remark that

$$
\begin{aligned}
& \sum_{j} \int_{(j-1) l}^{(j+1) l}|\Phi(x, i h)-\Phi(j l, i h)|^{2} d x \\
& \quad \leq \frac{\text { const }}{l} \sup \int_{(j-1) l}^{(j+1) l}|\Phi(x, i h)-\Phi(j l, i h)|^{2} d x \\
& \quad=\frac{\text { const }}{l}\|\Phi\|_{C_{0}^{\alpha}}^{2} \int_{(j-1) l}^{(j+1) l}|x-j l|^{2 \alpha} d x \\
& \quad=\text { const }\|\Phi\|_{C_{0}^{\alpha u}}^{2} l^{2 \alpha}
\end{aligned}
$$

and

$$
\sum_{j} \int_{(j-1) l}^{(j+1) l}\left|\left[\eta_{j}^{i}\right]\right|^{2} d x \leq \sum_{j} \int_{(j-1) l}^{(j+1) l}\left|\nabla \eta_{*}(\overline{\mathscr{U}})\left(\mathscr{U}_{-}^{i}-\mathscr{U}_{j}^{i}\right)\right|^{2} d x \leq \text { const } .
$$


So, if $p>2 /(1-\alpha)$,

$$
\left|L_{2}(\Phi)\right| \leq c\|\phi\|_{C_{0}^{\alpha}(\Omega)} l^{\alpha-1 / 2} \leq \mathrm{const}\|\Phi\|_{W_{0}^{1, p}(\Omega)} l^{\alpha-1 / 2} .
$$

Hence

$$
\left\|L_{2}\right\|_{W^{-1, q_{0}(\Omega)}} \leq \text { const } l^{\alpha-1 / 2} \underset{l \rightarrow 0}{\rightarrow} 0
$$

if $\alpha>\frac{1}{2}$ and $1<q_{0}<\frac{2}{1+\alpha}<2$. The proof of the theorem is complete.

Now we remark that we can find a family of probability measures $\left\{\nu_{(x, t)}\right\}_{(x, t) \in \Omega}$, the Young measures, associated to any sequence weak ${ }^{*}$-convergent in $L^{\infty}(\Omega)$, such that for any continuous function $H$ we have that $\left\{H\left(\mathscr{U}^{l}\right)\right\}$ converges weak ${ }^{*}$ to the value

$$
\int H(\lambda) \nu_{(x, t)}(d \lambda)
$$

By the previous results, applying the div-curl lemma (see [37]), we have the following Corollary 5.2. For any entropy-entropy flux pair $\left(\eta_{j}, q_{j}\right), j=1,2$, the commutation relation of Tartar, namely,

$$
\left\langle\nu_{(x, t)}, \eta_{1} q_{2}-\eta_{2} q_{1}\right\rangle=\left\langle\nu_{(x, t)}, \eta_{1}\right\rangle\left\langle\nu_{(x, t)}, q_{2}\right\rangle-\left\langle\nu_{(x, t)} \eta_{2}\right\rangle\left\langle\nu_{(x, t)}, q_{1}\right\rangle,
$$

holds.

In the following we establish strong convergence for the Lax-Friedrichs and the Godunov approximation.

Theorem 5.3. The Young measure $\nu$ is a point mass in the $\left(\omega_{-}, \omega_{+}\right)$-plane.

To prove this result we shall use a generalization (see also [19] and [31]) to our case of the theory developed by [34] for strictly hyperbolic systems. In fact, it is easy to show that the following properties hold.

Lemma 5.4. Let $\alpha$ be any number satisfying $\omega_{-}^{-}<\alpha<-\delta$, let $\varepsilon$ be such that

$$
\omega_{-}^{-}<\omega_{-}^{*}=\alpha-\varepsilon<\alpha<\omega_{-}^{\#}=\alpha+\varepsilon<-\delta,
$$

and let $(\eta, q)$ be of east type with limit $\omega_{-}^{*}$ and $(\bar{\eta}, \bar{q})$ be of west type with limit $\omega_{-}^{\#}$. Then the following conditions hold:

a) If, for all east-type entropy $\hat{\eta}$ with limit $\omega_{-}^{\#}$, we have $\langle\nu, \hat{\eta}\rangle=0$, then

$$
\operatorname{supp} \nu \cap\left\{\left(\omega_{-}, \omega_{+}\right): \omega_{-}^{\#} \leq \omega_{-} \leq-\delta, \omega_{+} \geq 0\right\}=\varnothing .
$$

b) Let $\theta_{-}$and $\bar{\theta}_{-}$be, respectively, the Goursat data for the entropies $\eta$ and $\bar{\eta}$ and suppose that their derivatives up to the second order valued at $\alpha$ are nonzero constants independent of $\varepsilon$. If, for all $\varepsilon>0$, one has

$$
\langle\nu, \eta \bar{q}-\bar{\eta} q\rangle=0,
$$

then

$$
\operatorname{supp} \nu \cap\left\{\left(\omega_{-}, \omega_{+}\right): \omega_{-}=\alpha, \omega_{+} \geq 0\right\}=\varnothing .
$$

c) There exists a constant $c$ independent of $\omega_{-}^{*}$ and of the entropy such that

$$
\langle\nu, q\rangle=c\langle\nu, \eta\rangle \text {. }
$$


d) If there exists an east-type entropy $\hat{\eta}$ with limit $\omega_{-}^{\#}$, so that $\langle\nu, \hat{\eta}\rangle \neq 0$, then for all west-type entropy pairs $\left(\bar{\theta}_{-}, \bar{q}\right)$ of limit $\omega_{-}^{\#}$, we have

$$
\langle\nu, \bar{q}\rangle=c\langle\nu, \bar{\eta}\rangle
$$

with the same constant $c$ corresponding to the entropies of east type with limit $\omega_{-}^{*}$, and satisfying the hypothesis (5.8).

Proof of Theorem 5.3. The reduction of the support of Young measures at a single point will be done in two steps. First, we show that it is concentrated at most in four points and then we reduce the support to a single point.

Let $\delta>0$ be the constant used to construct the entropies in Lemma 5.4, and suppose that $\omega_{-}^{-}<-\delta$. One has immediately

$$
\operatorname{supp} \nu \subseteq \mathscr{Q}_{-} \stackrel{\text { def }}{=}\left\{\left(\omega_{-}, \omega_{+}\right) \mid 0 \leq \omega_{+} \leq \omega_{+}^{+}, \omega_{-}=\omega_{-}^{-} \text {or }-\delta \leq \omega_{-} \leq 0\right\},
$$

and then

$$
\omega_{-}(\delta) \stackrel{\text { def }}{=} \inf \left\{y: \omega_{-}^{-} \leq y \leq-\delta, \operatorname{supp} \nu \cap\left\{\omega: y \leq \omega_{-}<-\delta\right\}=\varnothing\right\}=\omega_{-}^{-} .
$$

In fact, suppose $\omega_{-}(\delta)=-\delta$. By using Lemma 5.4 , we conclude that

$$
\operatorname{supp} \nu \cap\left\{\left(\omega_{-}, \omega_{+}\right): \omega_{-}=\alpha, \alpha \in\left(\omega_{-},-\delta\right)\right\}=\varnothing,
$$

which yields a contradiction. On the other hand, if $\omega_{-}(\delta) \in\left(\omega_{-}^{-},-\delta\right)$, we can show

$$
\operatorname{supp} \nu \cap\left\{\left(\omega_{-}, \omega_{+}\right): \omega_{-}=\alpha, \alpha \in\left(\omega_{-}, \omega_{-}(\delta)\right)\right\}=\varnothing ;
$$

so $\nu$ is concentrated on the lines $\omega_{-}=\omega_{-}(\delta), \omega_{-}=\omega_{-}^{-}$and the strip defined by $-\delta \leq \omega_{-} \leq 0$.

Let $\varepsilon>0$ be such that $\omega_{-}^{-}+\varepsilon<\omega_{-}(\delta),(\eta, q)$ be of east type with limit $\omega_{-}^{-}$and $(\bar{\eta}, \bar{q})$ of west type with limit $\omega_{-}^{-}+\varepsilon$. By Lemma 5.4 we have $\langle\nu, \eta \bar{q}-\bar{\eta} q\rangle=0$, for all $\varepsilon>0$. By the genuine nonlinearity of our system away from $\omega_{-}=0$, we get a contradiction as $\varepsilon \rightarrow 0$.

By a similar argument it follows that

$$
\operatorname{supp} \nu \subseteq \mathscr{Q}_{+} \stackrel{\text { def }}{=}\left\{\left(\omega_{-}, \omega_{+}\right) \mid \omega_{-}^{-} \leq \omega_{-} \leq 0, \omega_{+}=\omega_{+}^{+} \text {or } 0 \leq \omega_{+} \leq \delta\right\} ;
$$

so we obtain

$$
\operatorname{supp} \nu \subseteq \mathscr{Q}_{\delta} \stackrel{\text { def }}{=} \mathscr{Q}_{-} \cap \mathscr{Q}_{+}
$$

for all $\delta>0$. Then we have

$$
\operatorname{supp} \nu \subseteq\left\{(0,0),\left(\omega_{-}^{-}, 0\right),\left(0, \omega_{+}^{+}\right),\left(\omega_{-}^{-}, \omega_{+}^{+}\right)\right\} .
$$

By using now Theorem 6.1 in Serre [34] and because of the genuine nonlinearity of $(1.1)$ in the interior of $\mathscr{R}$, we can exclude one of the four corners of $\mathscr{R}$. The commutation relation of Tartar (5.7) and the mechanical energy, with the methods of [19] and [31], leads to showing that $\nu$ is a point mass. 
TheOREM 5.5. Under the hypotheses of Theorem 5.1, the approximate solutions $\left\{\left(u^{l}, v^{l}\right)\right\}$ converge (taking eventually a subsequence) strongly in $L_{\mathrm{loc}}^{p}, p<+\infty$, to a weak solution $(u, v)$ to system (1.1).

Proof. By using Theorem 5.3 and the fact that $v^{l}(x, t) \geq 0$, as proved in Theorem 5.1 , it follows that $\nu$ is a point mass in the $(u, v)$-plane. The theorem is thus proved.

Appendix. Riemann problem. In this section we consider the Riemann problem of the system (1.1) with data of the form

$$
(u, v)_{\left.\right|_{t=0}}=\left(u_{0}, v_{0}\right)= \begin{cases}\left(u_{-}, v_{-}\right) & x<0, \\ \left(u_{+}, v_{+}\right) & x>0\end{cases}
$$

where $v_{\mp} \geq 0$ and $u_{\mp}$ are constants. We shall solve this problem in the class of functions consisting of constant states, separated by either shock waves or rarefaction waves.

Next we discuss the construction of the shock-wave curves. Given a state $\left(u_{-}, v_{-}\right)$, we consider the possible states $(u, v)$ that can be connected to the state on the right by a back shock. The possible state $(u, v)$ must satisfy the Rankine-Hugoniot condition, which in this case reads

$$
\begin{aligned}
& \left(u-u_{-}\right) s=\left(\frac{1}{2}+n\right)\left(u^{2}-u_{-}^{2}\right)+f(v)-f\left(v_{-}\right), \\
& \left(v-v_{-}\right) s=u v-u_{-} v_{-} .
\end{aligned}
$$

Eliminating $s$ from these equations, we obtain

a) if $v_{-}>0$,

$$
\begin{aligned}
u-u_{-}= & \frac{v-v_{-}}{(2 n+1) v_{-}-(2 n-1) v} \\
& \times\left[2 n u_{-} \mp \sqrt{4 n^{2} u_{-}^{2}+2\left[(2 n+1) v_{-}-(2 n-1) v\right] \frac{f(v)-f\left(v_{-}\right)}{v-v_{-}}}\right]
\end{aligned}
$$

b) if $v_{-}=0$,

$$
v_{-}=0 \quad \text { or } \quad u-u_{-}=-\frac{1}{2 n-1}\left(2 n u_{-} \mp \sqrt{4 n^{2} u_{-}^{2}-2(2 n-1) f(v)}\right) .
$$

Thus, the set $\mathscr{S}$ of states that can be connected to $\left(u_{-}, v_{-}\right)$by a 1-shock on the right must lie on the curve $S_{1}=S_{1}\left(u_{-}, v_{-}\right)$;

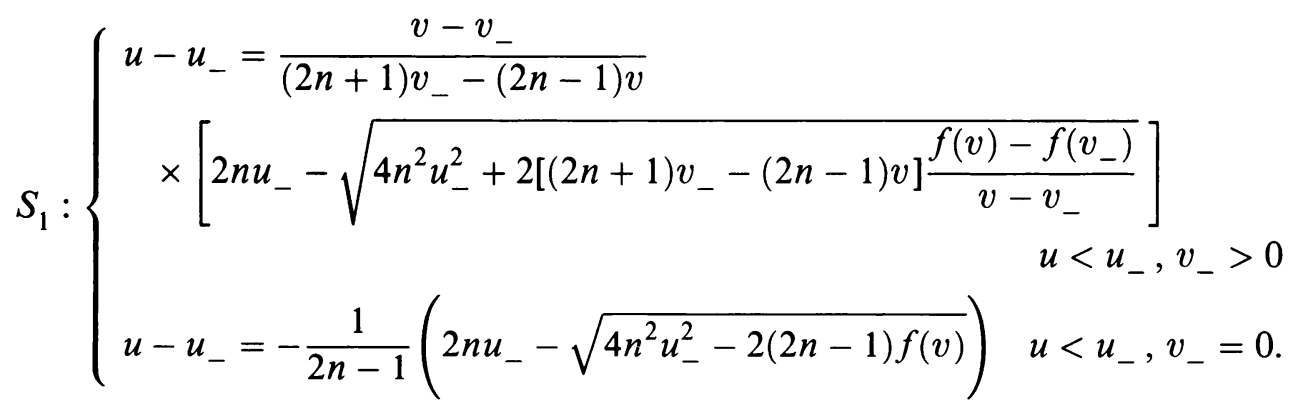


With a similar analysis, we can construct the curve $S_{2}$ consisting of all those states that can be connected to the state $\left(u_{-}, v_{-}\right)$by a 2 -shock on the right. We find the curve $S_{2}=S_{2}\left(u_{-}, v_{-}\right)$:

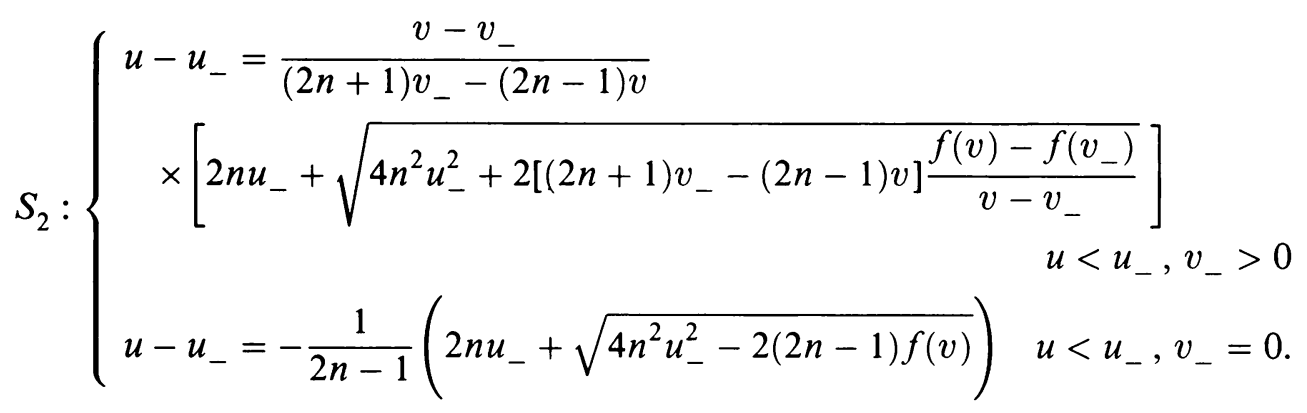

Now we recall that the integral curves have been studied in Sec. 2. So, the 1rarefaction wave curve $R_{1}=R_{1}\left(u_{-}, v_{-}\right)$is given by

$$
R_{1}:\left\{\begin{array}{l}
\left\{\begin{array}{l}
\frac{d v}{d u}=\frac{g_{-}(u, v)}{f^{\prime}(v)} \quad u>u_{-}, v_{-}>0 \\
v\left(u_{-}\right)=v_{-} \\
v=0 \quad u<u_{-}, v_{-}=0,
\end{array}\right.
\end{array}\right.
$$

while the 2-rarefaction wave curve $R_{2}=R_{2}\left(u_{-}, v_{-}\right)$is given by

$$
R_{2}:\left\{\begin{array}{l}
\left\{\begin{array}{l}
\frac{d v}{d u}=\frac{g_{+}(u, v)}{f^{\prime}(v)} \quad u>u_{-}, v_{-}>0 \\
v\left(u_{-}\right)=v_{-} \\
v=0 \quad u<u_{-}, v_{-}=0
\end{array}\right.
\end{array}\right.
$$

We can put all these curves together in the $(u, v)$-plane to obtain a diagram as in Fig. 1.

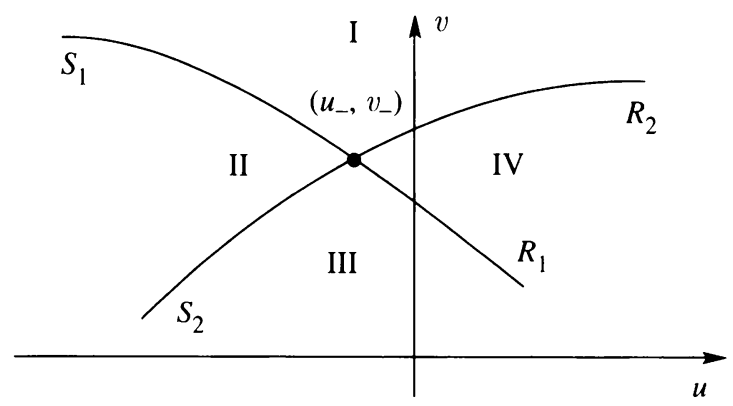

FIG. 1 
This shows that, for a fixed $\left(u_{-}, v_{-}\right)$, the $(u, v)$-plane is divided into four disjoint open regions I, II, III, IV. We remark that the curves $R_{1}$ and $S_{1}\left(R_{2}\right.$ and $S_{2}$ respectively) have second-order contact at $\left(u_{-}, v_{-}\right)[24,25]$.

Now consider the general Riemann problem (1.1)-(A.1). Take $\left(u_{-}, v_{-}\right)$as fixed and allow $\left(u_{+}, v_{+}\right)$to vary. If $\left(u_{+}, v_{+}\right)$lies on any of the above four curves, the problem is trivial.

We thus assume that $\left(u_{+}, v_{+}\right)$lies in one of the four open regions indicated in Fig. 1. We define, for a fixed $(\bar{u}, \bar{v}), W_{i}(\bar{u}, \bar{v})=S_{i}(\bar{u}, \bar{v}) \cup R_{i}(\bar{u}, \bar{v}), i=1,2$. Consider the point $(\bar{u}, \bar{v}) \in W_{i}\left(u_{-}, v_{-}\right)$such that $\left(u_{+}, v_{+}\right) \in W_{2}(\bar{u}, \bar{v})$. Then the solution to the Riemann problem (1.1)-(A.1) can be described as follows: we connect $(\bar{u}, \bar{v})$ to $\left(u_{-}, v_{-}\right)$on the right by a backward wave and then we connect $\left(u_{+}, v_{+}\right)$ to $(\bar{u}, \bar{v})$ on the right by a forward wave. The particular type of waves occurring depends, of course, on the position of $\left(u_{+}, v_{+}\right)$. In Fig. 2 (see p. 418) we illustrate the four various possibilities when $v \neq 0$. We observe that, in this case, the state $(\bar{u}, \bar{v})$ is such that $\bar{v}>0$, so that a vacuum (i.e., $v=0$ ) does not appear.

It remains to consider the case of a vacuum. We first consider $v_{-}>0$ and $v_{+}=0$. Referring to Fig. 1, we find the three possibilities illustrated in Fig. 3 (see p. 419). On the other hand, consider $v_{-}=0$ and $v_{+}>0$. We obtain in this case the two possibilities illustrated in Fig. 4 (see p. 420).

Using the results obtained in this section and the invariant regions result recalled at the end of Sec. 2, we obtain

THEOREM A.1. The Riemann problem (1.1)-(A.1) has a unique generalized solution in the class of two constant states separated by shocks and rarefaction waves, provided that $\left(u_{-}, v_{-}\right)$and $\left(u_{+}, v_{+}\right)$are sufficiently close. Finally, if the Riemann data lie in the invariant region (2.4) for some $c>0$, the solution of the corresponding Riemann problem also belongs to $\Sigma_{c}$. 


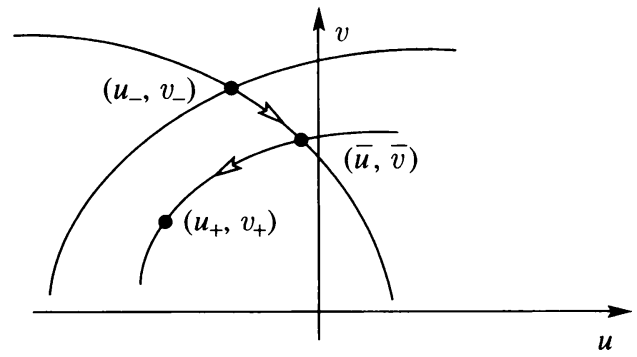

$\left(u_{+}, v_{+}\right)$in region $\mathrm{I}$

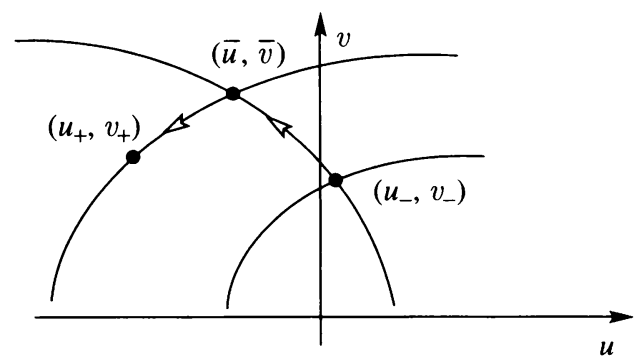

$\left(u_{+}, v_{+}\right)$in region $\mathrm{II}$

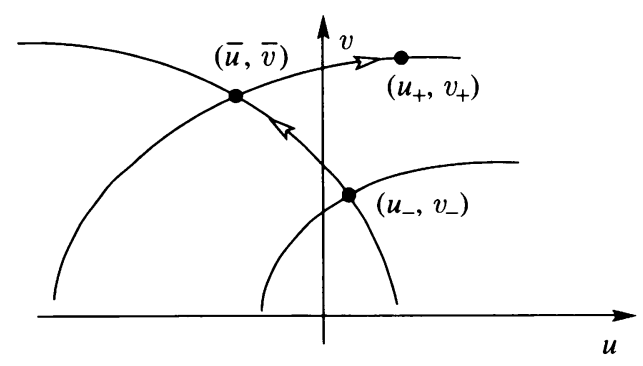

$\left(u_{+}, v_{+}\right)$in region III

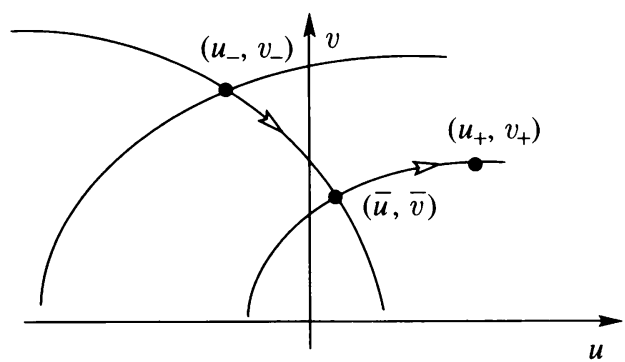

$\left(u_{+}, v_{+}\right)$in region IV
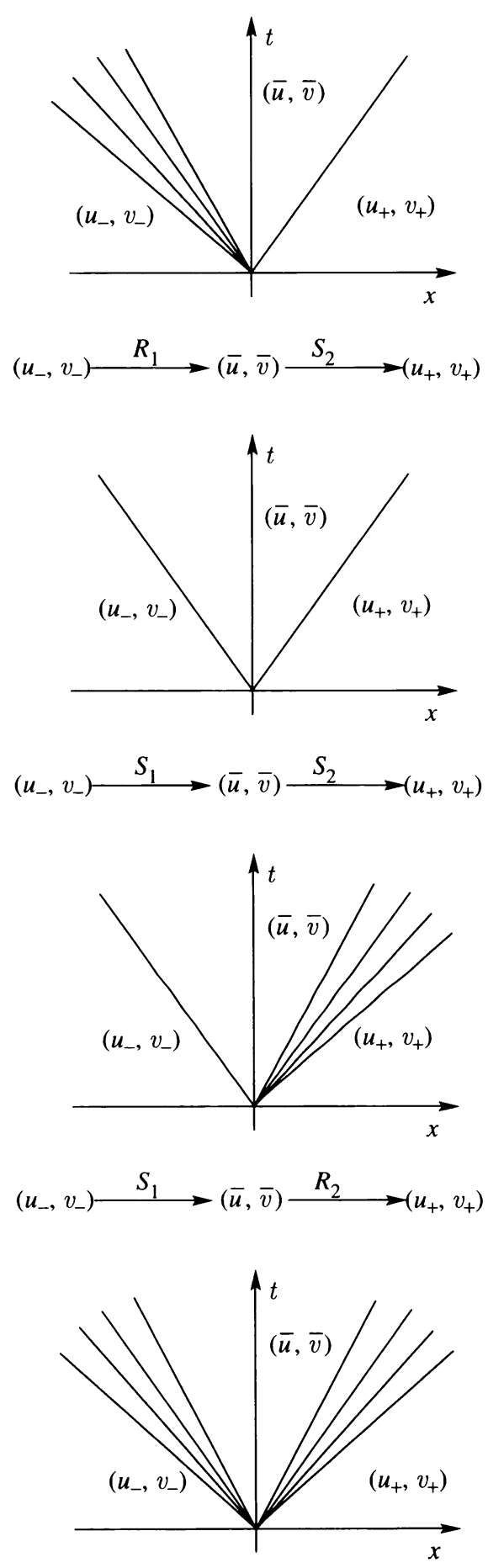

$$
\left(u_{-}, v_{-}\right) \stackrel{R_{1}}{\longrightarrow}(\bar{u}, \bar{v}) \stackrel{R_{2}}{\longrightarrow}\left(u_{+}, v_{+}\right)
$$

FIG. 2 


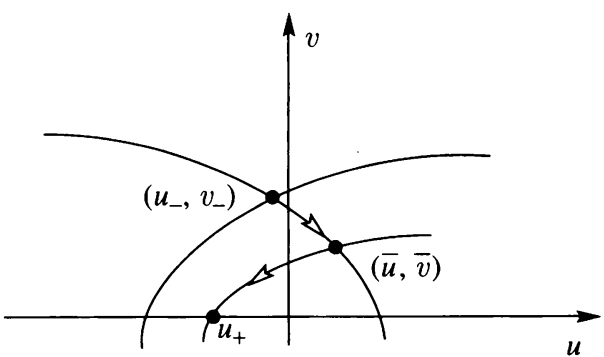

$\left(u_{+}, 0\right)$ in region I

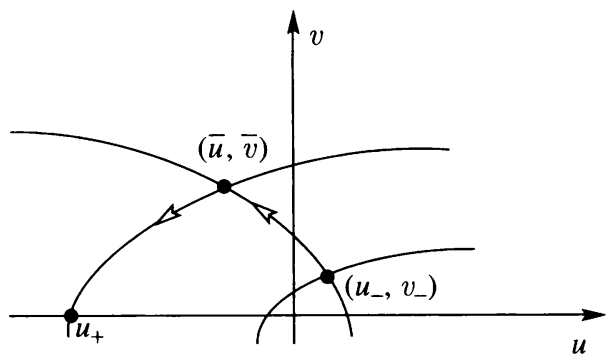

$\left(u_{+}, 0\right)$ in region II

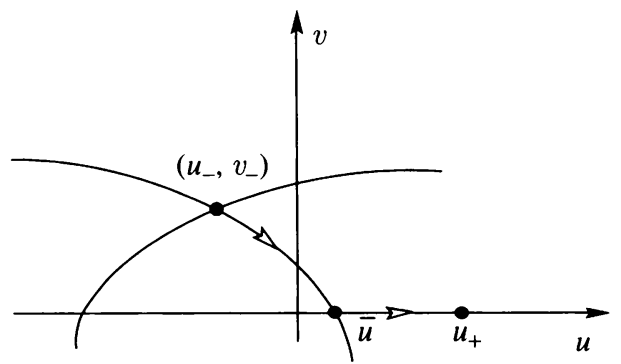

$\left(u_{+}, 0\right)$ in region IV
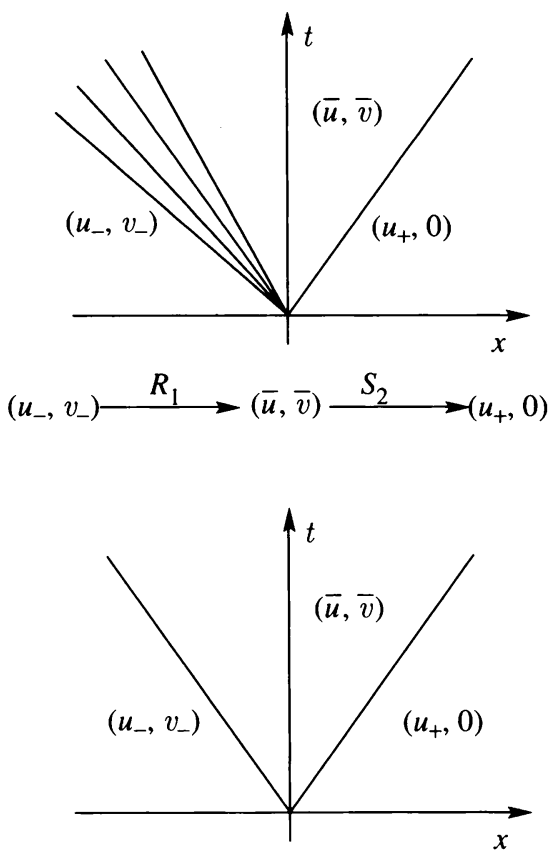

$\left(u_{-}, v_{-}\right) \stackrel{S_{1}}{\longrightarrow}(\bar{u}, \bar{v}) \stackrel{S_{2}}{\longrightarrow}\left(u_{+}, 0\right)$

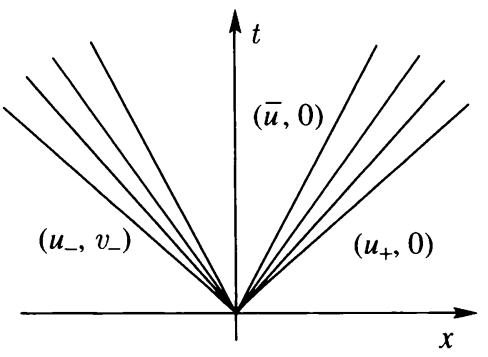

$\left(u_{-}, v_{-}\right) \stackrel{R_{1}}{\longrightarrow}(\bar{u}, 0) \stackrel{R_{2}}{\longrightarrow}\left(u_{+}, 0\right)$

Fig. 3 


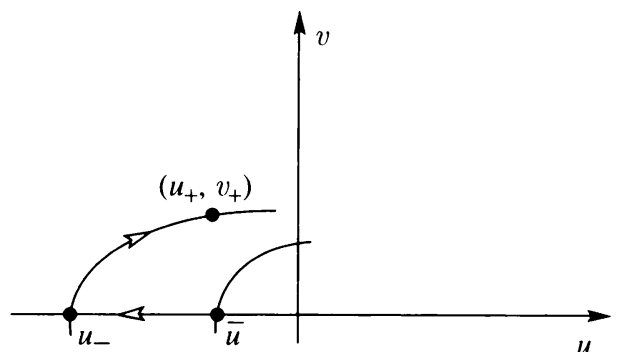

$\left(u_{+}, v_{+}\right)$in region III

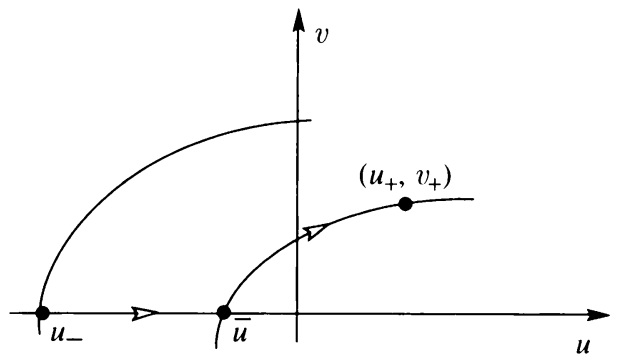

$\left(u_{+}, v_{+}\right)$in region IV
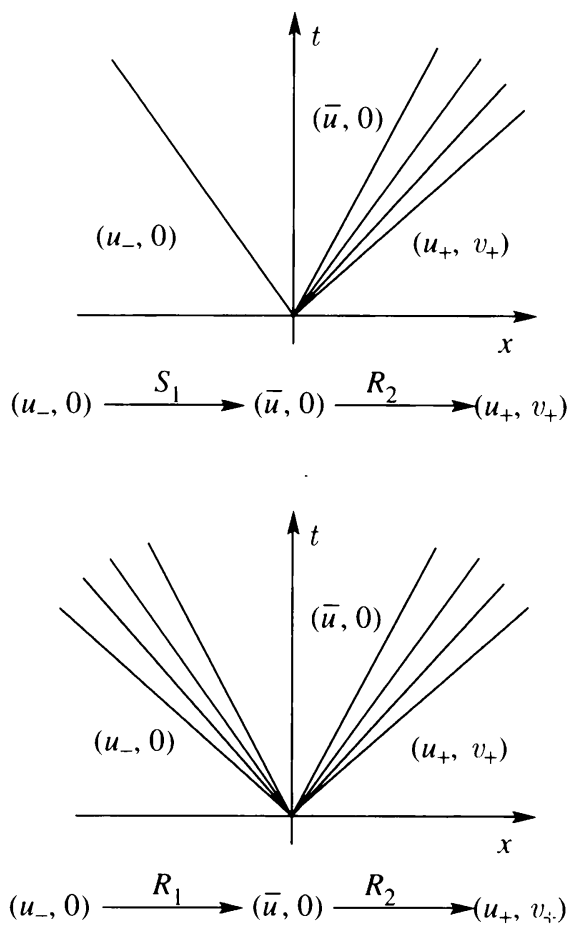

FIG. 4

\section{REFERENCES}

[1] G. Q. Chen, X. Ding, and P. Luo, Convergence of the Lax-Friedrichs scheme for isentropic gas dynamics I, Acta Math. Sci. 5, 415-432 (1985); II, Acta Math. Sci. 5, 433-472 (1985)

[2] G. Q. Chen, Convergence of the Lax-Friedrichs scheme for isentropic gas dynamics III, Acta Math. Sci. 6, 75-120 (1986)

[3] K. N. Chueh, C. C. Conley, and J. A. Smoller, Positively invariant regions for systems of non-linear diffusion equations, Indiana Univ. Math. J. 26, 372-411 (1977)

[4] R. Courant and D. Hilbert, Methods of mathematical physics II: Partial differential equations, Wiley and Sons, 1962

[5] M. G. Crandall and A. Majda, Monotone difference approximations for scalar conservation laws, Math. Comp. 34, 1-21 (1980)

[6] R. J. DiPerna, Compensated compactness and general systems of conservation laws, Trans. Amer. Math. Soc. 292, 383-420 (1985)

[7] R. J. DiPerna Convergence of approximate solutions to conservation laws, Arch. Rat. Mech. Anal. 82, 27-70 (1983)

[8] R. J. DiPerna, Convergence of the viscosity method for isentropic gas dynamics, Comm. Math. Phys. 91, 1-30 (1983)

[9] R. J. DiPerna, Measure-valued solutions to conservation laws, Arch. Rat. Mech. Anal. 88, 223-270 (1985)

[10] G. Glimm, The interaction of non-linear hyperbolic waves, Comm. Pure Appl. Math. 41, 569-590 (1988)

[11] S. K. Godunov, Difference method for the numerical computation of discontinuous solutions of equations of hydrodynamics, Mat. Sb. 47, 271-306 (1959)

[12] A. Harten, High resolution schemes for hyperbolic conservation laws, Jour. Comp. Phys. 49, 357-393 (1983) 
[13] A. Harten, J. M. Hyman, and P. D. Lax, On finite difference approximations and entropy conditions for shocks, Comm. Pure Appl. Math. 29, 297-322 (1976)

[14] A. Harten, P. D. Lax, and B. van Leer, On upstream differencing and Godunov-type schemes for hyperbolic conservation laws, SIAM Review 25, 35-61 (1983)

[15] D. Hoff, Invariant regions for systems of conservation laws, Trans. Amer. Math. Soc. 289, 591-610 (1985)

[16] L. Hörmander, Non-linear hyperbolic differential equations, Lectures Notes 1986-1987, Lund University, Sweden, 1988 (mimeographed notes)

[17] E. Isaacson, D. Marchesin, B. Plohr, and B. Temple, The Riemann problem near a hyperbolic singularity: The classification of solutions of quadratic Riemann problems I, SIAM Journal of Appl. Math. 48, 1009-1032 (1988)

[18] E. Isaacson and B. Temple, The classification of solutions of quadratic Riemann problems II, SIAM Journal of Appl. Math. 48, 1287-1301 (1988); III, SIAM Journal of Appl. Math. 48, 1302-1318 (1988)

[19] P.-T. Kan, On the Cauchy problem of a $2 \times 2$ system of non-strictly hyperbolic conservation laws, Ph.D. thesis, Courant Institute of Math. Sciences, N.Y. University, 1989

[20] B. L. Keyfitz and H. C. Kranzer, A system of non-strictly hyperbolic conservation laws arising in elasticity theory, Arch. Rat. Mech. Anal. 72, 219-241 (1980)

[21] S. Kružkov, First order quasi-linear equations with several space variables, Mat. Sb. 123, 228-255 (1970)

[22] P. D. Lax, Weak solutions of nonlinear hyperbolic equations and their numerical computation, Comm. Pure Appl. Math. 7, 159-193 (1954)

[23] P. D. Lax, Hyperbolic systems of conservation laws, II, Comm. Pure Appl. Math. 10, 537-566 (1957)

[24] P. D. Lax, Shock waves and entropy, in Contributions to Nonlinear Functional Analysis, ed. E. A. Zarantonello, Academic Press, 1971, pp. 603-634

[25] P. D. Lax, Hyperbolic systems of conservation laws and the mathematical theory of shock waves, SIAM, Philadelphia, 1973

[26] T. P. Liu, Zero dissipative limit for conservation laws, in Proceedings of the Conference on Non-linear Variational Problems and Partial Differential Equations, Isola d'Elba, 1990 (to appear)

[27] P. Marcati, Approximate solutions to the conservation laws via convective parabolic equations, Comm. Partial Differential Equations 13, 321-344 (1988)

[28] P. Marcati and A. Milani, The one-dimensional Darcy's law as the limit of a compressible Euler flow, J. Differential Equations, 129-147 (1990)

[29] F. Murat, Compacité par compensation, Ann. Scuola Normale Superiore Pisa 5, 489-507 (1978)

[30] I. G. Petrovskii, Partial Differential Equations, Iliffe Books, 1967

[31] B. Rubino, On the vanishing viscosity approximation to the Cauchy Problem for a $2 \times 2$ system of conservation laws, Ann. Inst. H. Poincaré Anal. Non Linéaire 10, 627-656 (1993)

[32] D. G. Schaeffer and M. Shearer, The classification of $2 \times 2$ systems of non-strictly hyperbolic conservation laws, with application to oil recovery, Comm. Pure Appl. Math. 40, 141-178 (1987)

[33] D. G. Schaeffer and M. Shearer, Riemann problems for non-strictly hyperbolic $2 \times 2$ systems of conservation laws, Trans. Amer. Math. Soc. 304, 267-306 (1987)

[34] D. Serre, La compacité par compensation pour les systèmes hyperboliques non linéaires de deux équations a une dimension d'espace, J. Math. Pures Appl. 65, 423-468 (1986)

[35] J. A. Smoller, Shock waves and reaction diffusion equations, Springer-Verlag, 1983

[36] S. L. Sobolev, Partial Differential Equations of Mathematical Physics, Pergamon Press, 1964

[37] L. Tartar, Compensated compactness and applications to partial differential equations, in Nonlinear Analysis and Mechanics: Heriott-Watt Symposium, IV, Research Notes in Math., 1979, pp. 136-210

[38] B. Temple, Global existence of the Cauchy problem for a class of $2 \times 2$ non-strictly hyperbolic conservation laws, Adv. Appl. Math. 3, 355-375 (1982) 